\title{
Normal Olfactory Discrimination Learning Set and Facilitation of Reversal Learning After Medial-Temporal Damage in Rats: Implications for an Account of Preserved Learning Abilities in Amnesia
}

\author{
H. Eichenbaum, A. Fagan, and N. J. Cohen \\ Department of Biological Sciences, Wellesley College, Wellesley, Massachusetts 02181, and \\ Department of Psychology, Johns Hopkins University, Baltimore, Maryland 21218
}

Recent evidence of preserved skill learning in patients with "global" amnesia has led to the postulation of a qualitative distinction between functionally separate memory systems, one of which may remain preserved when the other is profoundly impaired. On one account, the separate memory systems support either the learning of declarative knowledge, i.e., facts and associations, or the learning of procedural knowledge, i.e., knowledge that permits the expression of skilled performance without reference to specific facts or associations. In an effort to develop a rodent model of amnesia that illustrates the same distinction between memory systems, rats were trained in a series of discrimination and reversal problems using olfaction, a sensory modality in which they rapidly learn new associations. Rats with bilateral fornix, amygdala, or combined fornix and amygdala damage learned successive two-odor discriminations as quickly as normal and sham-operated control subjects.

Furthermore, all groups rapidly acquired the skills of discrimination as revealed in the development of a learning set. Subsequent presentation of a reversal of one discrimination elicited a marked dissociation among groups: Normal rats and rats with amygdala lesions required many more trials to acquire the reversal than to acquire a new discrimination problem, whereas rats with fornix lesions learned the reversal rather easily. A detailed analysis of response strategies suggested that normal rats and rats with amygdala lesions first extinguished the prior response tendencies and then abandoned the learning set skills and treated the reversal much as they did the initial discrimination problem. In contrast, after prior response tendencies were extinguished, rats with fornix lesions were relatively unaffected by the prior cue-associations and treated the reversal much as they would a new discrimination problem. These results distinguish between impaired and spared memory capacities in rodents with amnesia, in conformance with findings on human amnesia.

Until recently, investigators involved in developing animal models of amnesia have reported discrepancies in the phenomenology of behavioral impairment after comparable lesions in humans versus animals. Thus, whereas the memory disorder in human amnesic patients is remarkably pervasive, affecting many different categories of material, animals with lesions in the same brain structures suffer a rather more limited behavioral impairment. In particular, early experimenters were dismayed to

Received Apr. 29, 1985; revised Nov. 4, 1985; accepted Nov. 5, 1985.

This work was supported by grants NSF BNS 85-07677, PHS NS18744, and ONR N00014-83-K0037.

Correspondence should be addressed to Howard Eichenbaum, Department of Biological Sciences, Wellesley College, Wellesley, MA 02181.

Copyright (C) 1986 Society for Neuroscience $0270-6474 / 86 / 071876-09 \$ 02.00 / 0$ find that discrimination lcarning in such animals was normal even across long retention intervals (Douglas, 1967; Kimble, 1968; O’Keefe and Nadel, 1978).

More recent conceptualizations of amnesia, however, have begun to converge on a multicomponent view of memory and a less global view of amnesia, postulating fractionations among memory capacities and identifying distinct domains of impaired and spared memory capacities in amnesia. It is now clear that human amnesic patients with profound disorders of learning and memory have striking islands of normal memory. They can learn and retain normally a variety of skills and can show normal repetition priming effects, exhibiting facilitation in numerous performance tasks as a consequence of previous experience (for reviews, see Baddeley, 1982; Cohen, 1981, 1984; Schacter, 1985; Squire and Cohen, 1984; Tulving, 1985; Weiskrantz, 1982). Recent work with animals has been similarly directed at delineating domains of impaired and spared memory capacities after lesions of the hippocampus and related medial-temporal structures (Hirsh, 1974, 1980; Mishkin and Petri, 1984; Mishkin et al., 1984; O'Keefe and Nadel, 1978; Olton et al., 1979; Squire and Zola-Morgan, 1983; Winocur, 1980; Winocur and Olds, 1978). Whereas a total mapping between the human and the animal data has yet to be accomplished, the general acceptance by investigators working with human or animal amnesia of at least a phenomenological distinction between classes of memory capacities has constituted clear progress.

The present study examines further the separation of impaired and preserved learning capacities in rats and, more generally, inquires about the nature of the basic memory processes that support learning and remembering in this species. This work is designed in the context of proposals from the human amnesia literature hypothesizing dual memory systems (see citations above). The framework is accordingly a generic one, although it borrows particularly from Cohen and Squire's accounts of spared and impaired memory capacities (Cohen, 1981, 1984; Cohen and Squire, 1980; Squire and Cohen, 1984). Their work distinguishes between a procedural system, concerned with the acquisition and expression of skilled performance, and a declarative system, concerned with the accumulation and explicil remembering of facts and associations. Procedural memory is taken to effect modifications in perception and response to individual significant events by influencing the organization of the processing and action systems that guided performance in encountering such events. The changes in memory created by the procedural system are dedicated ones, expressible only through activation of the particular processing and action systems engaged by the original circumstances (or paradigm), remaining inaccessible outside of those circumstances (or paradigm). Declarative memory, by contrast, is taken to involve the creation 
of new data structures to represent relationships among multiple cues, within or across temporal frames, which have significance as the outcomes of particular processing experiences. The memory representations created by the declarative system are generally accessible to a variety of testing probes other than a literal re-presentation of the original circumstances and to a variety of processes, including those supporting recognition of the previous occurrence of the test stimuli. It is argued that amnesia results in the selective loss of the declarative memory system.

With this conceptual framework as background, our specific goals in the present experiments were (1) to bring into closer correspondence the testing procedures used with rodents and humans by selecting a specific sensory modality for which rats show the same special facility as humans do for vision, (2) to elucidate the properties of preserved and impaired discrimination learning in rats with medial-temporal damage within the framework of dual-memory-system theories, and (3) to evaluate the nature of amnesia in rats with different medial-temporal lesions. Each of these goals is discussed in turn, below.

\section{Olfaction as the test modality for studying learning and} memory in rats

Memory tests used to study humans and monkeys usually involve specific sensory modalities in which the subject rapidly acquires and easily retains specific information about the presented stimuli. Indeed, recent successes in the development of a primate model of amnesia have benefitted by basing the memory assessments on visual object memory, making use of what is probably their (and our) dominant sensory modality. In our attempt to provide memory testing materials that are truly analogous to those used for humans and monkeys, we here employ the olfactory mode, the dominant specific sensory modality of rodents. ${ }^{1}$ Previous work has demonstrated that the rate of acquisition of olfactory discrimination learning set in rats rivals that of visual learning set in humans and monkeys (Jennings and Keefer, 1969; Slotnick and Katz, 1974). Also, we have recently demonstrated, in an olfactory task analogous to that used for studies of visual object recognition in monkeys, excellent long-term recognition of odors sampled once briefly in normal rats plus marked amnesia in rats with medial-temporal damage (Eichenbaum et al., 1985).

We have focused on rats' dominant specific sensory modality because we suspect that, across mammalian species, processing in the dominant modality may encourage the rapid development of and may depend integrally on declarative representations. That is, comprehension of scenes and events invokes the dominant sensory modality to discriminate the various stimulus objects in a scene from the background, to identify the relationships among the various objects, and to appreciate their significance. Such processing demands would require a representational system that can maintain information about stimulus relationships (among stimuli within and across framcs; stimuli with respect to their spatial and temporal context). In our view, this is precisely the kind of information handled by the declarative memory system. This is not to suggest that declarative representations are not generated for and useful in other sensory modalities; indeed, any sensory modality that can support processing of multiple stimulus-object arrays will make use of declarative representation. However, the dominant sensory modality is most dependent on, and would place more immediate demands on, the declarative system because of the disproportionate role played by the dominant modality in processing stimulus relationships, and of the declarative memory system in storing them.

'The spatial mode is also a favorable one for rats, even in artificial laboratory conditions. However, this mode, in its unavoidable use of multiple cues, corresponds poorly to the specific sensory modes used in most testing with humans and primates.
Dissociation of preserved and impaired discrimination learning capacities

The present experiments took advantage of the superb olfactory system of rats to conduct a comprehensive examination of their learning and memory abilities both in the normal case and after medial-temporal damage, assessing discrimination learning, learning set, and reversal learning. Animals were trained on a series of odor discriminations in a manner that we believe invokes both memory systems. Specifically, use of the dominant sensory modality and exposure to a variety of paired stimuli and their differential reward values encourage the development of a representation of odor-pair relationships, that is, a declarative representation. At the same time, testing sensory discrimination learning with a continuous performance measure and making each discrimination independent of the identity (and, hence explicit remembering) of previous stimuli and reward assignments encourages the use of simple conditioning and extinction processes and generalization to common task rules, supported by a procedural representation. Either system would seem capable of supporting discrimination learning performance. Accordingly, in the absence of the declarative system, we expected amnesic rats to nonetheless perform well in odor discrimination learning and develop a discrimination learning set; and, indeed, we carefully selected task parameters on the basis of previous work (Kat et al., 1983; Staubli et al., 1984) that would permit a quantitatively normal rate of odor discrimination learning to occur in the animals with medial-temporal damage. It is notable that our strategy stands in contrast to that of previous investigations of discrimination learning using nonoptimal stimulus modalities and minimal pretraining. Those paradigms provide little opportunity to process multiple stimulus relationships, typically presenting only a single pair of stimuli for discrimination prior to the reversal. Accordingly, they place only minimal demands on the declarative system and can be solved solely on the basis of a procedural representation of discriminative stimuli in both normal and amnesic animals.

Our paradigm also offers an assessment of the extent to which the learning of amnesic and control groups shares the same basis. Following a set of discrimination problems, we evaluated the effects on both groups' performance of reversing the reward assignments of a previously learned odor discrimination. We expected the performance of normal animals to be harmed by the reversal because of explicit remembcring of thc odors' previous reward assignments, but expected performance of amnesic rats to be unharmed by the reversal because they would not recall the stimuli in their former context and would base their performance on the same memory processes used successfully to solve the earlier discrimination problems. With this particular paradigm, then, we expected a performance facilitation after hippocampal system damage, a finding that would provide particularly compelling evidence of the amnesic rats' reliance on a different memory system. This finding would contrast with previous observations of reversal impairment in other discrimination paradigms (cf. Kimble, 1968; O'Keefe and Nadel, 1978). Paradoxical findings of impairment in some task modalities and facilitation in another indicate that hippocampal system damage does not affect the reversal process itself. Rather, we suggest, such findings show that the reversal acts as a challenge to the form of representation in the original discrimination, revealing qualitatively distinct forms in normal and brain-damaged animals, depending on the task mode.

\section{Selective medial temporal lesions and amnesia}

Finally, this study addresses the issue of which medial-temporal structures are critical to memory in rats. Some recent studies have indicated that amnesia in monkeys and humans may follow only from combined damage to the hippocampus, or its 


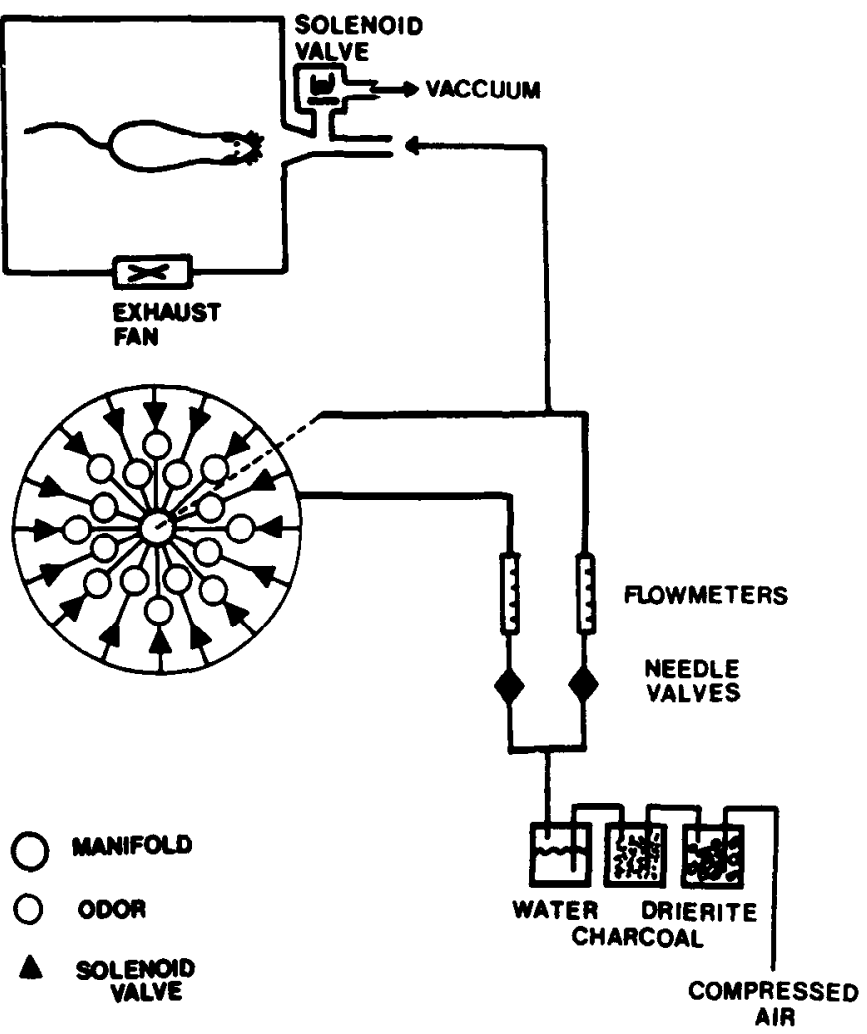

Figure 1. Schematic diagram of behavioral apparatus (top) and olfactometer (bottom).

pathways via the fornix, and the amygdala, or its pathways via the amygdalofugal system (Bachevalier et al., 1985; Mishkin, 1978). In order to examine the relative contributions of these brain systems in rodent memory processes, we compared the effects of separate and combined lesions of the fornix and amygdala on the performance of rats in the series of discrimination and reversal tasks described above. To evaluate the efficacy of our lesions in producing impairment on a task well known as sensitive to hippocampal system damage, the performance of subjects with combined fornix and amygdala lesions was also measured on spatial delayed alternation.

\section{Materials and Methods}

\section{Subjects}

Twenty-six adult male Long Evans hooded rats (Charles River) were maintained on a $23.75 \mathrm{hr}$ water-deprivation schedule with food available ad libitum. Surgery was performed on all operated subjects prior to behavioral training. The performance of a control group composed of normal $(n=5)$ and sham-operated $(n=2)$ rats was compared with that of animals with bilateral combination fornix and amygdala (FX + AMYG) lesions $(n=8)$, animals with bilateral fornix $(F X)$ lesions $(n-6)$, and animals with bilateral amygdala (AMYG) lesions $(n=5)$. Based on histological analyses, the behavioral data from two FX + AMYG and one FX animal were considered separately from those of the rest of their respective groups (see below).

\section{Surgery}

Rats were tranquilized with acepromazine and $45 \mathrm{~min}$ later anesthetized with sodium pentobarbital $(50 \mathrm{mg} / \mathrm{kg})$ and treated with atropine $(0.25$ $\mathrm{mg}$ ) to prevent excessive salivation. Body temperature was regulated using a $37^{\circ} \mathrm{C}$ isothermal pack. The rat's head was shaved and mounted in a Kopf stereotaxic device with bregma and lambda at equal height. Lesions were made by passing radio frequency current $(20 \mathrm{~V}$ for $30 \mathrm{sec})$ bilaterally at coordinate sites relative to bregma in the fornix (AP -1.3 ,
L 1.0, D 4.5) and in the amygdala (AP - 2.3, L 4.5, D 9.0). No lesions were made in the sham-surgery condition. The wound was closed with sutures, and the animal was allowed to recover at least 1 week before behavioral testing.

\section{Olfactory tasks}

The odor-discrimination apparatus (Fig. 1) consisted of a behavioral chamber connected to a flow-dilution olfactometer and microcomputer that controlled all presentation of stimuli, recorded behavioral responses, and presented rewards. The behavioral chamber was a $26.5 \times$ $25 \times 30 \mathrm{~cm}$ Plexiglas box enclosed in a sound-attenuating container with exhaust fan. The chamber included a conical sniff port on one wall and a water-reward cup located on the floor of the chamber just below the sniff port. Temporal presentation of the odors was controlled precisely by a specially designed stimulus control panel consisting of the sniff port with a photocell beam used to identify the presence of the nose in the port and a solenoid valve that could be switched either to present the stimulus into the port or to divert the odor to a vacuum exhaust dump. Odor stimuli were generated by a 16-channel, glass and Teflon olfactometer that mixed clean and odorized airstreams to $4 \%$ of saturation and presented the diluted odor to the stimulus control panel.

Rats were deprived of water for $48 \mathrm{hr}$ and shaped to poke their noses into the port in order to receive $0.05 \mathrm{ml}$ water rewards. Under program control, the rat was gradually shaped to maintain its nose in the port for $2 \mathrm{sec}$ to receive rewards. The program then added an $8 \mathrm{sec}$ intertrial interval (ITI), during which nose pokes did not provide reward, but instead delayed the end of the ITI by $2 \mathrm{sec}$. Availability of a new trial was signaled by a $100 \mathrm{~Hz}$ tone. Thereafter, nose entry into the port began the trial, as signaled by a $1 \mathrm{KHz}$ tone. Trials ended either when the nose was removed for $200 \mathrm{msec}(\mathrm{R}-)$ or when the $2 \mathrm{sec}$ nose poke response was completed $(R+)$. After 3-4 d of preliminary shaping, odor discrimination training began. Two odors, identified by humans as similar in class but easy to differentiate, were assigned arbitrarily as positive $(\mathrm{S}+)$ and negative $(\mathrm{S}-)$ stimuli. On a pseudorandom schedule, the $\mathrm{S}+$ or $\mathrm{S}$ - odor was presented as the nose poke initiated a trial. In this go/ no-go asymmetrically rewarded discrimination, animals were provided with reward only with completion of an $R+$ in the presence of the $S+$. Trials were presented until the subject reached a performance criterion of 18 correct out of 20 consecutive trials or 400 trials $/ \mathrm{d}$. The first discrimination was between $\mathrm{C} 14$-alkene and $\mathrm{C16}$-alkene, the second was between eugenol and guiacol, and the third was between coconut extract and almond extract. On the day after completion of the third discrimination, the odor valences for the third pair were reversed and the stimuli were presented as a new discrimination problem. On the next day after reversal, a fourth discrimination problem was presented. The odor stimuli on this problem were citronellyl butyrate and citronellyl acetate.

\section{Spatial delayed alternation}

Training was accomplished in a Y-maze composed of three $10 \mathrm{~cm}$ wide and $1 \mathrm{~m}$ long arms, with $8 \mathrm{~cm}$ high walls and a Plexiglas top. The arms were positioned in equiangular orientation. Two goal arms had $1 \mathrm{~cm}$ deep reward cups at their ends. After familiarization with the maze, 48 $\mathrm{hr}$ water-deprived rats were rewarded with $0.1 \mathrm{ml}$ water only on trials in which they alternated arm choice. Correction trials were administered after errors. Trials were given at $60 \mathrm{sec}$ intertrial intervals for 50 trials/d until a performance criterion of 18 correct out of 20 consecutive trials was reached or a maximum of 600 trials was presented. All normal and FX + AMYG subjects were trained on spatial alternation after completion of olfactory testing; rats from other groups were not trained or tested on this task.

\section{IIistology}

After training, rats were anesthetized deeply with an overdose of sodium pentobarbital and perfused transcardially with $0.9 \%$ saline and then $10 \%$ formol-saline. Heads were removed and stored in $10 \%$ formalin. The brain was then removed and stored in 30\% sugar formalin for $3 \mathrm{~d}$. Fifty-micron sections were taken in the coronal plane. Every third section was mounted and stained with cresylecht violet. Adjacent sections were prepared with myelin stain (Loyez method) to visualize fiber damage. Lesions wcre reconstructed using the Pellingro and Cushman atlas (1967). 


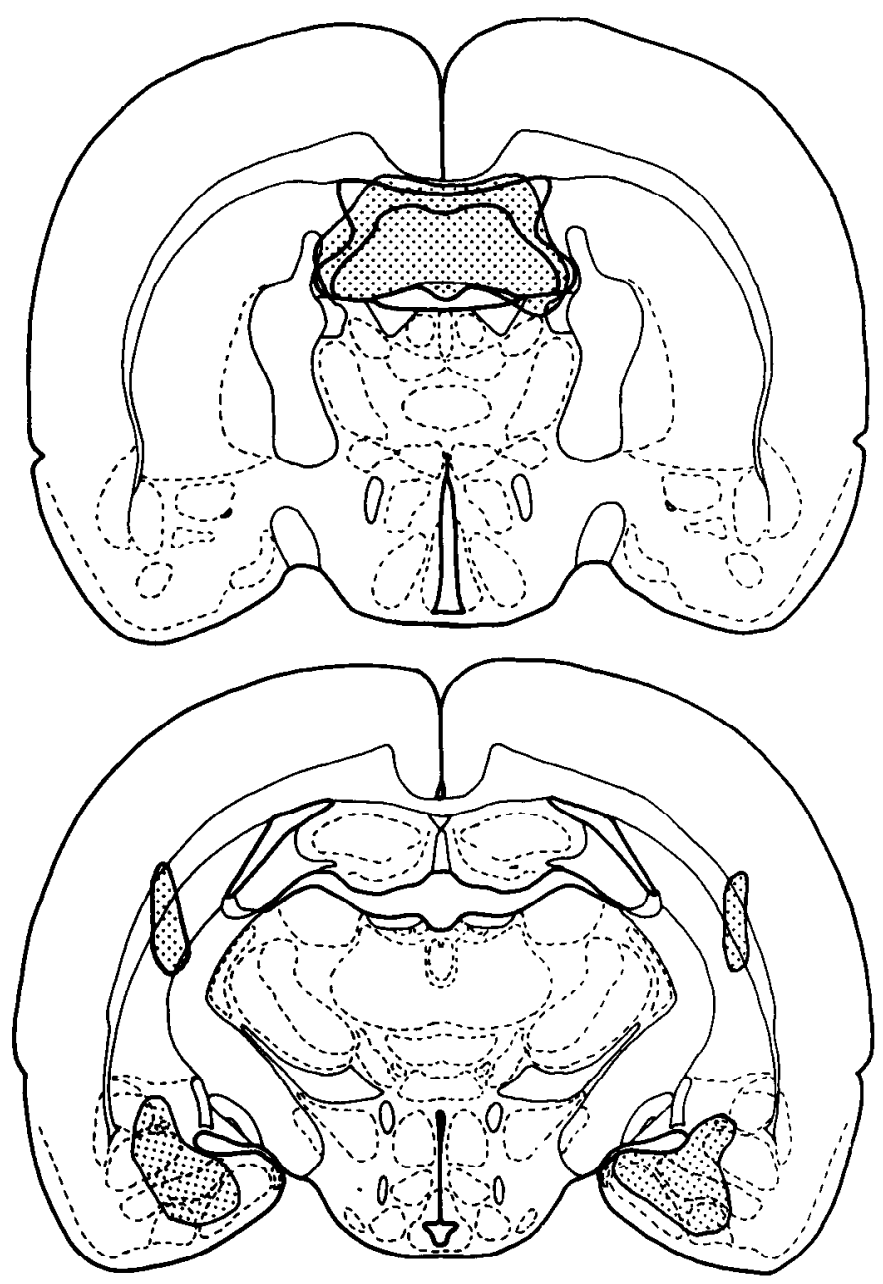

Figure 2. Reconstructions of coronal sections through the center of average-sized ablations of fornix (top) and amygdala (bottom).

\section{Results}

\section{Histology}

Figure 2 shows representative reconstructions of each lesion type. Fornix lesions produced considerable, although not always complete, destruction in that structure. In a few subjects, additional damage was found in the septal nucleus and rostral pole of the hippocampus, as well as overlying neocortex and corpus callosum. In three cases (two FX + AMYG and one FX), there was bilateral damage in the mediodorsal thalamus. Amygdala lesions were confined to amygdaloid nuclei; the lateral and medial basal nuclei were consistently ablated. The three subjects whose brain lesions included bilateral damage in the mediodorsal thalamus required considerably more trials than all other animals on the initial discrimination task and are considered separately from the other animals in reporting our data.

\section{Olfactory tasks}

Figure 3 shows the performance of all groups in odor discrimination and reversal problems. Comparisons of group performance indicated that brain lesions did not affect odor discrimination or the ability to acquire an odor discrimination set, but did markedly affect performance in the reversal problem. Analysis of variance demonstrated no significant differences across subject groups $(F=2.66 ; d f=3 / 19, p>0.1)$ but did reveal highly significant differences in scores across problems $(F=$ 51.94, $d f=4 / 76, p<0.001$ ) and in group differences in scores across problems $(F=2.69, d f=12 / 76, p<0.01)$.

\section{Odor discrimination learning}

Group mean scores on the first discrimination ranged from 233 to 310 trials. Post hoc Scheffé comparisons revealed no significant group differences in acquisition of the first, or any other, specific discrimination problem $(p>0.2)$.

\section{Discrimination learning set}

Discriminations subsequent to the first were learned much more rapidly, ranging in group mean from 27 to 78 trials, demonstrating similar and successful learning set in all groups. Scheffè analyses indicated that all groups required significantly fewer trials to reach criterion in discriminations two to four compared to the first (all $p<0.01$ ). Performance among discriminations two to four did not differ significantly, indicating that the acquisition of learning set was largely complete by the end of the first discrimination task.

\section{Reversal learning}

Group performances differed strikingly when subjects were challenged with the reversal. Control and AMYG subjects took many more trials to acquire the reversal than they took to acquire the original discrimination of the same odors in the third discrimination problem (both $p<0.01$ ). Indeed, they required about as many trials to complete the reversal as needed to learn the initial discrimination problem (i.e., scores for the reversal were not significantly different from scores for the first discrimination, both $p>0.2$ ). Furthermore, for these groups combined, rank ordering of individuals' performance on the reversal and on the initial discrimination were well correlated by linear regression $(r=0.93, F=59.4, d=1 / 10, p<0.0001$; see Fig. 4). Performance on the reversal was not well correlated with performance on the discrimination of the same stimuli (i.c., the third discrimination problem; $r=-0.24, F=0.62, d f=1 / 10$, $p>0.2$ ).

Both FX + AMYG and FX subjects required many fewer trials for the reversal than both the AMYG and control groups (all $p<0.01$ ). In contrast to the performance of the AMYG and control groups, reversal for the FX or FX+AMYG groups did not require a significantly greater number of trials than acquisition of the immediately prior (third) discrimination problem $(p>0.2)$, although every animal showed some increase in trials to criterion. The performance of individuals in the FX and FX+AMYG groups on the reversal problem did not correlate significantly with their performance on the initial discrimination problem $(r=-0.42, F=1.98, d f=1 / 9, p>0.2$; see Fig. 4), or on the third discrimination problem $(r=-0.05, F=0.03$, $d f=1 / 9, p>0.2$ ).

\section{Analysis of learning stages}

Further analyses of the data were performed in an effort to clarify the nature of the reversal facilitation in the FX and FX+AMYG groups. These analyses attempted to reveal the sequence of strategies or "hypotheses" animals employed during the course of training within problems (Krechevsky, 1935). In our previous work with the present kind of olfactory learning tasks (Eichenbaum et al., 1980, 1983), we found that most errors that occur early in discrimination learning are errors of commission, i.e., production of $\mathrm{R}+$ during presentation of $\mathrm{S}-$. This is not surprising given that rewards are provided only for the positive response $(R+$ during presentation of $S+)$. Thus, we expected in this experiment, too, that animals would employ a "Response" hypothesis, producing mostly $\mathrm{R}+$ responses regardless of stimulus type. Later in training, we expected the appearance 


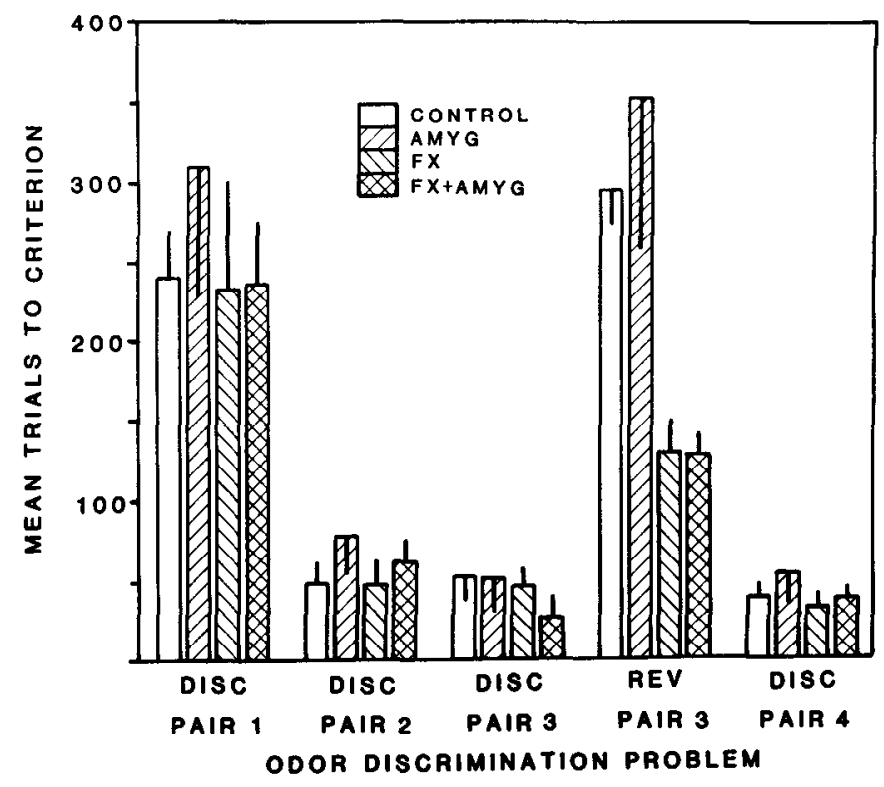

Figure 3. Performance of control and brain-damaged rats on discrimination $(D I S C)$ and reversal $(R E V)$ problems with successive pairs of odors. Groups are identified by locus of bilateral lesion: CONTROL, normal and sham-operated; $A M Y G$, amygdala; $F X$, fornix; $F X+A M Y G$ combination fornix and amygdala. Vertical bars indicate standard error.

of $R-$ to $S-$, while maintaining $R+$ to $S+$, revealing the adoption of a "Correct" hypothesis. Furthermore, we expected that normal rats would begin the reversal with a long sequence of incorrect responses to both stimuli, revealing an "Incorrect" hypothesis consistent with the former stimulus valences. After that stage in the reversal, we expected performance similar to that during a discrimination problem, i.e., responding consistent with Response and then Correct hypotheses.

Stages of performance were operationally defined and measured by observing whether each response was consistent with one of the above described hypotheses. Our technique used a "sliding window" to observe the sequence of responses following each successive trial. A trial was considered a Response hypothesis trial if it was the first of a block of trials containing 15 $\mathrm{R}+$ responses out of 20 consecutive trials $(p<0.02$ in the binomial distribution), regardless of stimulus presented. It was considered a Correct hypothesis trial if it was the first of a block of trials containing 15 correct $(S+R+$ or $S-R-)$ responses out of 20 consecutive trials. It was considered an Incorrect hypothesis trial if it was the first of a block of trials containing 15 incorrect $(S+R-$ or $S-R+)$ responses out of 20 consecutive trials. Hypothesis stages were subsequently defined as continuous blocks of trials that began with five sequential trials of a single hypothesis and ended with the last block in that hypothesis. Successive stages could overlap. Pcrformance by stages for all groups is shown in Figure 5.

The first discrimination was characterized for all groups by a long Response stage followed by a relatively short Correct stage before reaching criterion. Subsequent discriminations were characterized by a markedly reduced Response stage but a relatively constant Correct stage. No Incorrect stage was observed in any animal on any discrimination problem. The reversal was characterized by the appearance of a short Incorrect stage in all groups, followed by the reappearance of a prolonged Response stage selectively in the Control and AMYG groups compared to that in the FX + AMYG and FX groups, then finally a slightly, but not significantly, increased Correct stage for the Control and AMYG groups.

Analyses of variance and appropriate Scheffé tests were em-

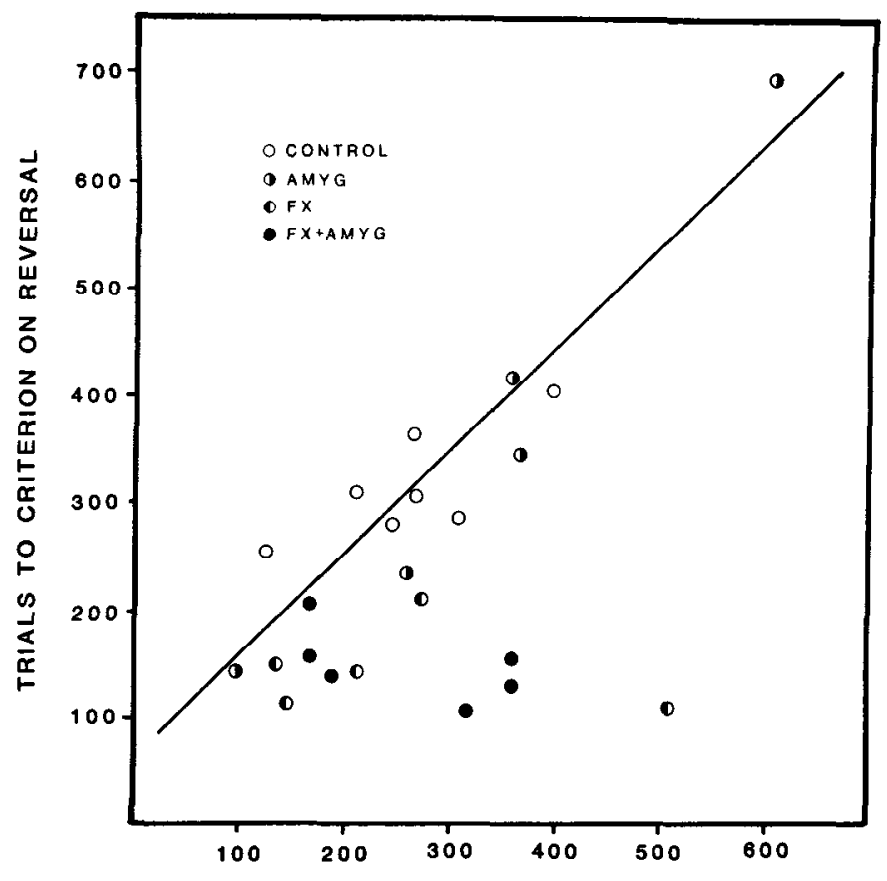

TRIALS TO CRITERION ON INITIAL DISCRIMINATION

Figure 4. Prediction of reversal score by performance on the initial odor-discrimination problem. Individual scores of all subjects are plotted with respect to the linear-regression estimate generated for the combined Control and AMYG groups. Note that reversal scores for all FX and FX + AMYG subjects fall lower than that predicted by their initial discrimination performance according to the regression line. (The authors thank David Olton for suggesting this illustration.)

ployed to compare the performance of groups across problems for each separate stage of learning. Group comparisons of performance in the Response stage paralleled that of the trials-tocriterion measure. Thus, analysis of variance revealed highly significant problem differences $(F=37.61, d f=4 / 76, p<0.001)$ and group $\times$ problem interaction $(F=2.29, d f=12 / 76, p<$ $0.01)$. Scheffe analyses indicated that this stage was longer in the first discrimination than in all subsequent discrimination problems for all groups (all $p<0.05$ ). In the reversal, both the Control and AMYG groups took significantly more trials in the Response stage than both the FX + AMYG and FX groups (all $p<0.02$ ). The Control and AMYG groups required significantly more trials to complete the Response stage in the reversal task than they did in the immediately prior (third) discrimination (both $p<0.01$ ); FX + AMYG and FX groups did not (both $p>$ 0.2 ). Finally, neither the Control nor the AMYG group required a significantly different number of trials to complete the $\mathrm{Re}$ sponse stage of the reversal problem than to complete that stage of the initial discrimination (both $p>0.2$ ); the FX+AMYG and FX groups took many fewer trials in the Response stage of the reversal (both $p<0.01$ ).

Analysis of variance of the Correct stage revealed an overall significant problem difference $(F=2.75, d f=12 / 76, p<0.05)$, apparcntly reflecting the small increase in trials required for the reversal problem, but no significant group differences $(F=0.58$, $d f=3 / 19, p>0.2$ ).

Most of the subjects in each group met our criteria for an Incorrect stage at the beginning of the reversal problem, the only problem for which this stage was observed. The mean number of trials in this stage did not vary significantly among groups. In order to increase our confidence in these Incorrect stage findings, we conducted one final analysis. Because $R-$ responses during $\mathbf{S}+$ are relatively rare in our asymmetrically rewarded 
discrimination tasks, we took this error of maintaining former cue assignments during reversal to be a significant event and limited our analysis to its occurrences. We counted the number of $\mathbf{S}+\mathbf{R}$ - responses in the first 20 trials of the reversal, when we expected the occurrence of such responses to be greatest. The mean number of $S+R-$ trials rose for all groups during reversal compared to that in the prior discrimination as revealed by a significant problem difference in the analysis of variance $(F=10.52, d f=12 / 76, p<0.001)$; but no significant group differences were observed on this measure $(F=1.34, d f=3 / 19$, $p>0.2$ ).

\section{Spatial delayed alternation}

In contrast to their superior performance in the odor-discrimination reversal task, FX + AMYG subjects were severely impaired in the spatial delayed alternation. Two of the FX+AMYG subjects did not reach the performance criterion in 600 trials and were assigned that score. The mean score of FX+AMYG subjects was 411.0 trials. The mean score of the normal Control subjects (with the exception of one that became ill and could not be trained) was 99.5 trials. No normal subject required as many trials as any FX + AMYG subject to acquire the task. A $t$ test revealed that the $F X+A M Y G$ group required significantly more trials to reach criterion than the group of four normal Control subjects $(t=3.32, d f=8, p<0.01)$.

\section{Discussion}

\section{Locus of damage needed to produce amnesia}

In the present study, rats with medial-temporal system damage exhibited the expected amnesic pattern of sparing and loss of memory across the several tasks. The subtotal combined fornix and amygdala lesions, which produced severe impairment in spatial delayed alternation, resulted in a normal rate of odordiscrimination learning and marked facilitation of odor-discrimination reversal. Before considering the nature of this pattern of performance and its implications for theories of memory and amnesia, some observations can be offered about which medial-tcmporal structures are critical for producing amnesia. The performance of rats with FX+AMYG damage was indistinguishable from that of rats with FX damage; they both demonstrated the predicted pattern of amnesic performance, in contrast to the performance of control animals and of animals with AMYG damage. Hence, fornix damage was necessary and sufficient to produce the amnesic profile.

From his studies of monkeys, Mishkin (1978) concluded that combined lesions of the hippocampus and amygdala are required for global amnesia; his work suggests that combined lesions produce much greater memory deficits than damage restricted to hippocampus or amygdala alone (also see Zola-Morgan and Squire, 1984b). Other investigators, however, have found significant impairments in monkeys following lesions of the fornix or hippocampus alone (Gaffan, 1974, 1977; Mahut et al., 1982; Moss et al., 1981). The degree of preoperative experience and age at operation may be important considerations in resolving this discrepancy (Mahut and Moss, 1984), as might be details of operative procedure (Zola-Morgan and Squire, 1984b). In rats, it is clear that damage to the hippocampus, or indeed its output pathway, the fornix, produces major learning and memory impairments (e.g., Green and Stauff, 1974; Jarrard, 1980; Olton et al., 1982). The present results are consistent with the findings for rats; in addition, they suggest that for this species, lesions that include the amygdala are no more effective than lesions of the hippocampal pathway alone.

In the present study, three animals had inadvertent bilateral damage in the mediodorsal thalamus (see Histology). Their performance was unlike that of the amnesic rats with fornix damage in that they showed a deficit in the basic odor-discrimination

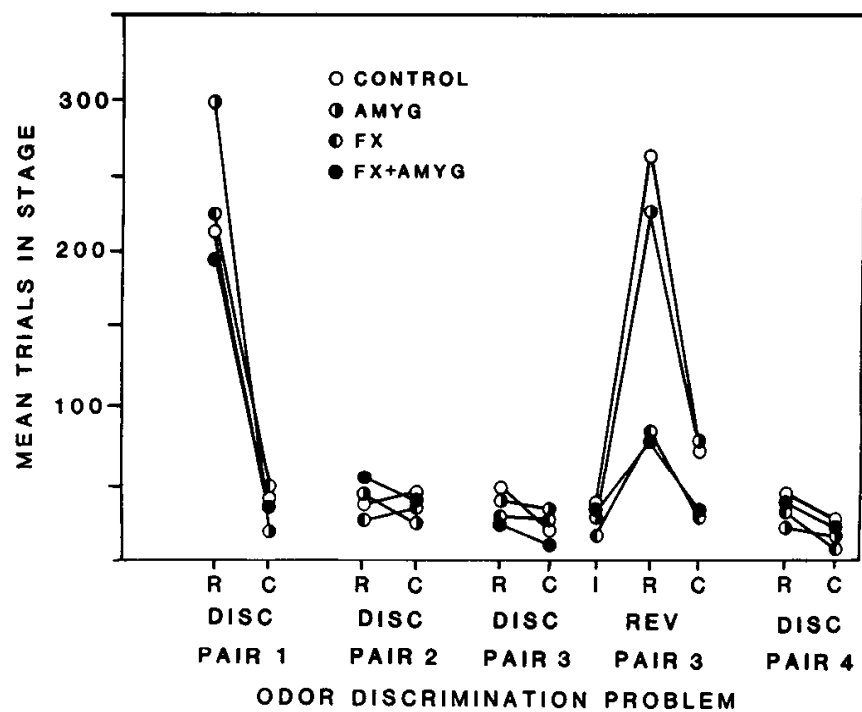

Figure 5. Performance of control and brain-damaged rats on successive stages in discrimination $(D I S C)$ and reversal $(R E V)$ problems with successive pairs of odors. See text for description of stages: $R$, Response stage; $C$, Correct stage; $I$, Incorrect stage. See Figure 3 for designation of groups.

task. This deficit is consistent with previous observations of such impairments in animals with damage to structures in the olfactory thalamocortical pathway (Eichenbaum et al., 1980; Sapolsky and Eichenbaum, 1980).

\section{Preserved sensory discrimination}

Rats with fornix lesions learned several odor discriminations as rapidly as normal animals. This result is consistent with a number of previous reports of successful discrimination learning despite damage to the hippocampal system (Kimble, 1963; Kimble and Zack, 1967; Malamut et al., 1984; Murphy and Brown, 1974; Samuels, 1972; Schmaltz and Theios, 1972; Silveira and Kimble, 1968; Teitelbaum, 1964; Webster and Voneida, 1964; Winocur and Mills, 1970; Winocur and Olds, 1978), but is in conflict with other reports of impaired discrimination learning following hippocampal systcm damagc (Becker and Olton, 1979; Hirsh, 1974; Jarrard, 1975; Kimble, 1963; Myhrer and Kaada, 1975; O'Keefe and Nadel, 1978; Olton et al., 1980; Winocur, 1979, 1980; Winocur and Mills, 1970). In our attempt to reconcile this discrepancy, we can offer the following empirical generalization consistent with the procedural/declarative framcwork: Studies revealing impairment fall into three rough categories. Hirsh's and Winocur's studies on contextual influences either make the appropriateness of the critical cues conditional on the presence of other cues (Hirsh) or test the effects of manipulating the background cues on the discriminability of the relevant cues (Winocur). The spatial tasks (including spatial alternation) make the reward value of a given arm in a maze dependent on its relationship to some distal cues (O'Keefe and Nadel) and/or to previous responses (Olton et al.). Finally, complex maze tasks (Myhrer and Kaada) make the choices at any given choice-point dependent on memory for its position with respect to the other choice points. In all of these tasks, successful performance places a premium on representing relational information, a memory demand for which we argue the declarative system is particularly well suited; amnesic rats, without recourse to the declarative system, show discrimination learning impairments in all of these tasks. By contrast, impairments are not seen after medial-temporal damage in discrimination learning tasks for which successful performance does not depend on 
direct comparison among stimuli and in which the significance of stimuli remains constant, as in sensory discrimination problems involving the visual, tactile, olfactory, and gustatory modalities (citations above) and in simple conditioning paradigms in animal (Schmaltz and Theios, 1972) and human amnesia (Weiskrantz and Warrington, 1979).

Other investigators have proposed somewhat similar accounts of spared and impaired discrimination learning abilities in animals with medial-temporal damage. Zola-Morgan and Squire (1984a) explained sensory discrimination learning and amnesia within the procedural/declarative framework, arguing that discrimination learning involved two distinct components: a "gradual tuning in" of the critical stimulus dimension that distinguishes among the stimuli, supported by the procedural memory system, and learning and remembering which stimuli are associated with reward, supported by the declarative system. They argued that discrimination learning always involved both components but could favor one over the other depending on task parameters and that amnesic animals could show either normal or impaired discrimination performance (also see ZolaMorgan and Squire, 1985). Olton et al. (1979) claimed a preservation of "reference memory," noting the ability of amnesic animals to learn the constant significance of cues over repeated trials, in contrast to a deficient "working memory," specialized for keeping track of the temporary status of changing circumstances. Hirsh (1974) argued that preserved learning occurred along the "performance line" connecting the sensory represention of the stimulus to observable response by associative mechanisms proposed in S-R theory. He argued that amnesia resulted from a deficit in retrieval of memory for stimuli in relationship to the "context" of other cues. Mishkin and Petri (1984) adopted Hirsh's explanation, referring to preserved discrimination ability as "habit" formation as opposed to impaired "memory" for contexl-dependent information. O'Keefe and Conway (1980) demonstrated that rats with fornix damage were impaired in a spatial discrimination task dependent on the use of multiple distributed cues but were unimpaired in the task when the same cues were concentrated near the goal. They interpreted these data as showing an impaired capacity for "place" learning, to be distinguished from a spared capacity for "cue" learning (for a more detailed description, see O'Keefe and Nadel, 1978). We suggest that impaired learning and memory after medial-temporal damage occur for all tasks requiring remembering of relational information, whether it is the spatial relationships among cues, the relationships among critical cues and their temporal context, or other relationships among cues. Tasks that can be solved by learning directly the significance of individual cues, as in the present experiment, remain preserved.

\section{Preserved skill learning}

The present experiment additionally demonstrates that discrimination learning set is preserved following damage to medialtemporal structures. Harlow (1959) characterized set learning as the acquisition of the rule of the task, usually "win-stay, loseshift," and as the elimination of nonrewarding strategies. Restle (1958) suggested that the improvement was due to "tuning in" to common qualities of the stimuli across specific discriminations. Whether one of these is the appropriate description of what animals learned in the present study, or whether they learned some other unspecified category of information, cannot yet be determined. Future studies demonstrating significant positive transfer across discriminations with extradimensional shifts in the stimulus would provide evidence for response rule learning; studies demonstrating greater positive transfer across discriminations involving intradimensional shifts would provide evidence for the learning of stimulus rules (see Schwartz et al., 1971). Until such results are forthcoming, we shall continue to use the phrase "learning set" to refer to positive transfer ob- tained across discrimination problems, without prejudice about the underlying mechanisms. We suggest that preserved acquisition of learning set by rats with medial-temporal damage in the present experimental paradigm constitutes an example of preserved skill learning and corresponds to the spared acquisition of perceptual and cognitive skills in amnesic humans and monkeys (also see Zola-Morgan and Squire, 1984a).

\section{Facilitation of reversal learning}

Perhaps the most important finding of the present study is the superiority of the amnesic rats over the controls in learning the reversal problem. We had predicted reversal facilitation at the outset of this study based on expectations of qualitatively different representations of discriminative stimuli by control versus amnesic rats. These expectations appear to be borne out by the present data, as is perhaps best illustrated by the analysis of learning stages (Incorrect, Response, and Correct) for the reversal task. In the Incorrect stage, animals faced with the same stimuli as on the previous day continue for a time to produce the same responses as were appropriate on the previous day; such performance provides a clear indication of $24 \mathrm{hr}$ retention in amnesic animals, although these data alone cannot permit us to determine whether such retention is mediated by a preserved memory system or the residual capacity of an impaired system.

Performance in the Response stage, by contrast, is more revealing. The data here are consistent with our proposal that normal rats and rats with fornix damage were guided by different kinds of memory representations. For controls, performance on the reversal problem and the very first discrimination problem were highly correlated in a subject-by-subject analysis, each requiring a nearly equivalent number of trials for the group to learn, and each characterized by a nearly equivalently prolonged (compared to the second and third discrimination problems) Response stage. The reversal performance of normal rats thus may be seen as an abandonment of previously successful odordiscrimination strategies and a return to strategies adopted during the original discrimination learning. In the context of our framework, we suggest that this reaction was due to a conflict betwecn their cxplicit remcmbering of former assignments of odors to differential reward and the perceived absence of differential reward during their Incorrect stage responding. By contrast, performance on the reversal problem for rats with fornix damage was poorly correlated with, and was learned in many fewer trials than, the first discrimination problem; this was due primarily to the much shorter Response stage in the reversal problem than in the first discrimination problem. The Response stage in the reversal problem was much like what the amnesic animals exhibited in the second and third discrimination problems. Thus, the reversal performance of rats with fornix damage seems best characterized as similar to acquisition of just another discrimination problem in the set, due to amnesia for the declarative information that played so important a role in the performance of the control subjects. The fact that it did take longer for amnesic rats to learn the reversal problem than it did for them to learn the previous discrimination is likely due to the need to "extinguish" responding to stimuli previously learned as individually significant. The existence of a significant Incorrect stage provides evidence for this interpretation.

The facilitation of reversal following hippocampal system damage, though predicted, stands in contrast to many observations of reversal impairment reported in the literature. This apparent contradiction can be understood by considering the differences between the paradigms used previously and the present one within our dual-memory-system framework. Hirsh (1974) used a framework somewhat similar to ours to offer an account of reversal impairment in those previous paradigms. He argued that reversal performance of animals with hippocampal system damage would be characterized by two stages of learning-ex- 
tinction then acquisition - thus taking them about twice the number of trials to reverse as was required for acquisition of the original discrimination. Normal rats, however, would have access to an additional memory system not available to those with hippocampal system damage. This memory system, even if irrelevant to performance on the previous discrimination problem, would permit representation of the relationship between old and current cue-reward contingencies, allowing rapid reversal to occur without successive extinction-then-acquisition stages. In our framework, the declarative system would be capable of representing just such information, conferring a large advantage on control animals in such tasks.

Comprehensive review of the literature seems to provide at least general support for this notion. We found seven studies with complete data in which animals with hippocampal system damage first performed normally on a sensory discrimination in a task using specific sensory cues (the appropriate comparison for the present study) and then were impaired on its reversal (Berger and Orr, 1982; Nonneman and Isaacson, 1973; Samuels, 1972; Silveira and Kimble, 1968; Teitelbaum, 1964; Webster and Voneida, 1964; Winocur and Olds, 1978). As was expected in our account, the amnesic animals' impairment across the studies could be traced to the fact that control animals were bothered little by the reversal, requiring only $0.8-2.5 \times$ the number of trials to learn it to criterion as they required to learn the original discrimination; in fully five of those studies, the ratio of trials to learn the reversal versus trials to learn the original discrimination was less than 2.0. By contrast, in the present study, where amnesic animals showed facilitation rather than impairment of reversal learning, control animals had a ratio of 6.0 and 7.1, for Control and AMYG groups, respectively. This suggests that control animals were performing in a very different manner in the present study. Specifically, in our view we take the difference in outcome to be a function of the extent to which declarative memory contributes to the representation of discriminative stimuli prior to reversal. In the only other published findings of reversal facilitation, testing involved highly trained subjects performing within their dominant sensory modality, visual objects in monkeys (Zola and Mahut, 1973) and odors in rats (Kat et al., 1983; Staubli et al., 1984). In the present task, rats were trained with a number of odor-discrimination problems prior to reversal. We suggest that either the cue modality or training sophistication, or both, determine the predominant representational strategies, favoring the use of the declarative system in normal but not amnesic subjects.

A complete account of the paradoxical facilitation of reversal learning in animals with fornix damage will depend on future empirical studies using the present, or other, theoretical formulations to make specific (and, hopefully, quantitative) a priori predictions of outcome in normal and amnesic animals. Nonetheless, the present studies provide clear evidence of preserved discrimination learning and learning set in rats with medialtemporal damage, and failure of the preserved memory system to produce the normal pattern of performance in reversal learning. These results conform with predictions based on a dualmemory-system framework offered originally to account for the phenomenology of human amnesia; the observed correspondence in the qualities of preserved and impaired memory capacities in the amnesia of humans and rats provides further support for this general framework.

\section{References}

Bachevalier, J., J. P. Aggleton, and M. Mishkin (1985) Visual recognition in monkeys: Effects of separate vs. combined transection of the fornix and the amygdalofugal pathways. Exp. Brain Res. 57: 554 561.

Baddeley, A. (1982) Amnesia: A minimal model and an interpretation.
In Human Memory and Amnesia, L. Cermak, ed., Erlbaum, Hillsdale, NJ.

Becker, J. T., and D. S. Olton (1979) Object discrimination by rats: The role of frontal and hippocampal systems in retention and reversal. Physiol. Behav. 24: 33-38.

Berger, T. W., and W. B. Orr (1982) Role of the hippocampus in reversal learning of the rabbit nictitating membrane response. In Conditioning: Representation of Involved Neural Functions, C. D. Woody, ed., Plenum, New York.

Cohen, N. J. (1981) Neuropsychological evidence for a distinction between procedural and declarative knowledge in human memory and amnesia. Ph.D. thesis, University of California, San Diego.

Cohen, N. J. (1984) Preserved learning capacity in amnesia: Evidence for multiple memory systems. In The Neuropsychology of Memory, N. Butters and L. R. Squire, eds., Guilford, New York.

Cohen, N. J., and L. R. Squire (1980) Preserved learning and retention of a pattern-analyzing skill in amnesia: Dissociation of knowing how and knowing that. Science 210: 207-210.

Douglas, R. J. (1967) The hippocampus and behavior. Psychol. Bull. 67: 416-442.

Eichenbaum, H., K. J. Shedlack, and K. Eckmann (1980) Thalamocortical mechanisms in olfaction. I. Effects of lesion of the mediodorsal thalamic nucleus and frontal cortex on olfactory discrimination in the rat. Brain Behav. Evol. 17: 255-275.

Eichenbaum, H., R. A. Clegg, and A. Feeley (1983) Reexamination of functional subdivisions of the rodent prefrontal cortex. Exp. Neurol. 79: 434-451.

Eichenbaum, H., T. Parikh, and N. Cohen (1985) Delayed non-match to sample with trial unique odor stimuli in intact and fornix-damaged rats: A new test for recognition memory and model of temporal lobe amnesia. Abstr. Soc. Neurosci. 11: 333.

Gaffan, D. (1974) Recognition impaired and association intact in the memory of monkeys after transection of the fornix. J. Comp. Physiol. Psychol. 86: 1100-1109.

Gaffan, D. (1977) Monkeys' recognition memory for complex pictures and the effect of fornix transection. Q. J. Exp. Psychol. 29: 505-514.

Green, E., and C. Stauff (1974) Behavioral role of hippocampal connections. Exp. Neurol. 45: 141-160.

Harlow, H. L. (1959) Learning set and error factor theory. In Psychology: A Study of a Science, S. Koch, ed., McGraw-Hill, New York.

Hirsh, R. (1974) The hippocampus and contextual retrieval of information from memory: A theory. Behav. Biol. 12: 421-444.

Hirsh, R. (1980) The hippocampus, conditional operations and cognition. Physiol. Psychol. 8: 175-182.

Jarrard, L. (1975) The role of interference and retention by rats with hippocampal lesions. J. Comp. Physiol. Psychol. 89: 400-408.

Jarrard, L. (1980) Selective hippocampal lesions and behavior. Physiol. Psychol. 8: 198-206.

Jennings, J. W., and L. H. Keefer (1969) Olfactory learning set in two varieties of domestic rat. Psychol. Rep. 24: 3-15.

Kat, A. G., H. Eichenbaum, and N. J. Cohen (1983) Olfactory discrimination and reversal learning in rats with combined hippocampus-amygdala lesions. Abstr. Soc. Neurosci. 9: 638.

Kimble, D. P. (1963) The effects of bilateral hippocampal lesions in rats. J. Comp. Physiol. Psychol. 56: 273-283.

Kimble, D. P. (1968) Hippocampus and internal inhibition. Psychol. Bull. 70: 285-295.

Kimble, D. P., and S. Zack (1967) Olfactory discrimination in rats with hippocampal lesions. Psychon. Sci. 8: 211-212.

Krechevsky, I. (1935) Brain mechanisms and "hypotheses." J. Comp. Psychol. 19: 425-462.

Mahut, H., and M. Moss (1984) Consolidation of memory: The hippocampus revisited. In The Neuropsychology of Memory, N. Butters and L. R. Squire, eds., Guilford, New York.

Mahut, H., S. Zola-Morgan, and M. Moss (1982) Hippocampal resections impair associative learning and recognition memory in the monkey. J. Neurosci. 2: 1214-1229.

Malamut, B. L., R. C. Saunders, and M. Mishkin (1984) Monkeys with combined amygdalo-hippocampal lesions succeed in object discrimination learning despite 24 -hour retention intervals. Behav. Neurosci. 98: 759-769.

Mishkin, M. (1978) Memory in monkeys severely impaired by combined but not separate removal of amygdala and hippocampus. Nature 273: 297-298.

Mishkin, M., and H. L. Petri (1984) Memories and habits: Some 
implications for the analysis of learning and retention. In The Neuropsychology of Memory, N. Butters and L. R. Squire, eds., Guilford, New York.

Mishkin, M., B. Malamut, and J. Bachevalier (1984) Memories and habits: Two neural systems. In The Neurobiology of Learning and Memory, J. L. McGaugh, G. Lynch, and N. Weinberger, eds., Guilford, New York.

Moss, M., H. Mahut, and S. Zola-Morgan (1981) Concurrent discrimination learning of monkeys after hippocampal, entorhinal, or fornix lesions. J. Neurosci. 1: 227-240.

Murphy, L. R., and T. S. Brown (1974) Hippocampal lesions and learned taste aversion. Physiol. Psychol. 2: 60-64.

Myhrer, T., and B. Kaada (1975) Locomotor avoidance and maze behavior in rats with dorsal fornix transected. Physiol. Behav. 14: 847-853.

Nonneman, A. J., and R. L. Isaacson (1973) Task dependent recovery after early brain damage. Behav. Biol. 8: 143-172.

O'Keefe, J., and D. H. Conway (1980) On the trail of the hippocampal engram. Physiol. Psychol. 2: 229-238.

O'Keefe, J., and L. Nadel (1978) The Hippocampus as a Cognitive Map, Oxford U. P., Oxford, UK.

Olton, D. S., J.T. Becker, and G. E. Handleman (1979) Hippocampus, space, and memory. Brain Behav. Sci. 2: 313-365.

Olton, D. S., J. T. Becker, and G. E. Handleman (1980) Hippocampal function: Working memory or cognitive mapping. Physiol. Psychol. 8: 239-246.

Olton, D. S., J. A. Walker, and F. H. Gage (1982) A disconnection analysis of hippocampal function. Brain Res. 233: 241-253.

Pellingro, L. J., and A. J. Cushman (1967) A Stereotaxic Atlas of the Rat Brain, Appleton-Century-Crofts, New York.

Restle, F. (1958) Toward a quantitative description of learning set data. Psychol. Rev. 65: 77-91.

Samuels, I. (1972) Hippocampal lesions in the rat: Effects on spatial and visual habits. Physiol. Behav. 8: 1093-1098.

Sapolsky, R. M., and H. Eichenbaum (1980) Thalamocortical mech anisms in odor-guided behavior. II. Effects of lesions of the mediodorsal thalamic nucleus and frontal cortex on odor preferences and sexual behavior in the hamster. Brain Behav. Evol. 17: 276-290.

Schacter, D. L. (1985) Priming of old and new knowledge in amnesic patients and normal subjects. Ann. NY Acad. Sci. 444: 41-530.

Schmaltz, L. W., and J. Theios (1972) Acquisition and extinction of a classically conditioned response in hippocampectomized rabbits. J. Comp. Physiol. Psychol. 79: 328-333.

Schwartz, R. M., M. Schwartz, and R. C. Tees (1971) Optional intradimensional and extradimensional shifts in the rat. J. Comp. Physiol. Psychol. 77: 470-475.

Silveira, J. C., and D. P. Kimble (1968) Brightness discrimination and reversal in hippocampally-lesioned rats. Physiol. Behav. 3: 625-630.
Slotnick, B. M., and H. M. Katz (1974) Olfactory learning set formation in rats. Science 185: 796-798.

Squire, L. R., and N. J. Cohen (1984) Human memory and amnesia. In Neurobiology of Learning and Memory, G. Lynch, J. McGaugh, and N. Weinberger, eds., Guilford, New York.

Squire, L. R., and S. Zola-Morgan (1983) The neurology of memory: The case for correspondence between the findings for man and nonhuman primate. In The Physiological Basis of Memory, J. A. Deutsch, ed., Academic, New York.

Staubli, U., G. Ivy, and G. Lynch (1984) Hippocampal denervation causes rapid forgetting of olfactory information in rats. Proc. Natl. Acad. Sci. USA 81: 5885-5887.

Teitelbaum, H. (1964) A comparison of the effects of orbitofrontal and hippocampal lesions upon discrimination learning and reversal in the cat. Exp. Neurol. 9: 452-462.

Tulving, E. (1985) How many memory systems are there? Am. Psychol. 40: 385-398.

Webster, D. B., and T. J. Voneida (1964) Learning deficits following hippocampal lesions in split-brain cats. Exp. Neurol. 10: 170-182.

Weiskrantz, L. (1982) Comparative aspects of studies of amnesia. Phil. Trans. R. Soc. Lond. 298: 97-109.

Weiskrantz, L., and E. K. Warrington (1979) Conditioning in amnesic patients. Neuropsychologia 17: 187-194.

Winocur, $G$. (1979) The effects of interference on discrimination learning and recall by rats with hippocampal lesions. Physiol. Behav. 22: 339-345.

Winocur, G. (1980) The hippocampus and cue utilization. Physiol. Psychol. 8: 280-288.

Winocur, G., and J. A. Mills (1970) Transfer between related and unrelated problems following hippocampal lesions in rats. J. Comp. Physiol. Psychol. 73: 162-169.

Winocur, G., and J. Olds (1978) Effects of context manipulation on memory and reversal learning in rats with hippocampal lesions. $J$. Comp. Physiol. Psychol. 92: 312-321.

Zola, S., and H. Mahut (1973) Paradoxical facilitation of object reversal learning after transection of the fornix in monkeys. Neuropsychologia 11: 271-284.

Zola-Morgan, S., and L. R. Squire (1984a) Preserved learning in monkeys with medial temporal lesions: Sparing of motor and cognitive skills. J. Neurosci. 4: 1072-1085.

Zola-Morgan, S., and L. R. Squire (1984b) Performance of monkeys with separate and combined lesions of the hippocampus and amygdala on delayed nonmatching to sample. Soc. Neurosci. Abstr. 10: 385.

Zola-Morgan, S., and L. R. Squire (1985) Medial temporal lesions in monkeys impair memory on a variety of tasks sensitive to human amnesia. Behav. Neurosci. 99: 22-34. 